Research Article

\title{
Network Pharmacology and Molecular Docking Suggest the Mechanism for Biological Activity of Rosmarinic Acid
}

\author{
Minglong Guan $\mathbb{D}^{1,2,3}$ Lan Guo $\mathbb{D}^{4},{ }^{4}$ Hengli $M a \mathbb{D}^{1,2,3}$ Huimei $W u\left(\mathbb{D},{ }^{1,2,3}\right.$ \\ and Xiaoyun Fan 1 1,2,3 \\ ${ }^{1}$ Department of Geriatric Respiratory and Critical Care, The First Affiliated Hospital of Anhui Medical University, Jixi Road 218, \\ Hefei, Anhui 230022, China \\ ${ }^{2}$ Anhui Geriatric Institute, Jixi Road 218, Hefei, Anhui 230022, China \\ ${ }^{3}$ Anhui Key Laboratory of Geriatric Molecular Medicine, Anhui Medical University, Jixi Road 218, Hefei, Anhui 230022, China \\ ${ }^{4}$ Department of Biochemistry and Molecular Biology, School of Basic Medical Sciences, Anhui Medical University, \\ Meishan Road 81, Hefei, Anhui 230032, China
}

Correspondence should be addressed to Xiaoyun Fan; 13956988552@126.com

Received 1 July 2020; Revised 24 February 2021; Accepted 30 March 2021; Published 12 April 2021

Academic Editor: Susana Gorzalczany

Copyright $\odot 2021$ Minglong Guan et al. This is an open access article distributed under the Creative Commons Attribution License, which permits unrestricted use, distribution, and reproduction in any medium, provided the original work is properly cited.

Rosmarinic acid (RosA) is a natural phenolic acid compound, which is mainly extracted from Labiatae and Arnebia. At present, there is no systematic analysis of its mechanism. Therefore, we used the method of network pharmacology to analyze the mechanism of RosA. In our study, PubChem database was used to search for the chemical formula and the Chemical Abstracts Service (CAS) number of RosA. Then, the Traditional Chinese Medicine Systems Pharmacology Database and Analysis Platform (TCMSP) was used to evaluate the pharmacodynamics of RosA, and the Comparative Toxicogenomics Database (CTD) was used to identify the potential target genes of RosA. In addition, the Gene Ontology (GO) enrichment analysis and Kyoto Encyclopedia of Genes and Genomes (KEGG) pathway enrichment analysis of target genes were carried out by using the web-based gene set analysis toolkit (WebGestalt). At the same time, we uploaded the targets to the STRING database to obtain the protein interaction network. Then, we carried out a molecular docking about targets and RosA. Finally, we used Cytoscape to establish a visual protein-protein interaction network and drug-target-pathway network and analyze these networks. Our data showed that RosA has good biological activity and drug utilization. There are 55 target genes that have been identified. Then, the bioinformatics analysis and network analysis found that these target genes are closely related to inflammatory response, tumor occurrence and development, and other biological processes. These results demonstrated that RosA can act on a variety of proteins and pathways to form a systematic pharmacological network, which has good value in drug development and utilization.

\section{Introduction}

Traditional Chinese medicine and natural compounds contain a large number of active components. This provides more possibilities and opportunities for the development and the use of drugs. Rosmarinic acid (RosA) is one of the water-soluble natural phenolic acid compounds [1]. It is widely found in Labiatae and Arnebia plants such as Rosmarinus officinalis, Melissa officinalis, and Hyssopus cuspidatus Boriss [2]. Increasing studies have shown that RosA not only has strong antiinflammatory activity but also has antibacterial, antivirus, and antitumor activity [3-7]. Moreover, RosA showed antiallergic inflammation, antifibrosis, and liverprotective effects [8-10]. So RosA has been paid more attention in the fields of pharmacy, food, cosmetics, and so on. However, the molecular mechanism induced by RosA and the corresponding cell phenotypic changes have not been systematically studied. At the same time, the use of computational methods to identify and predict drug targets and potential mechanisms is becoming a major method $[11,12]$. This approach cannot only speed up the process of drug discovery and design but also save money, time, and energy. 
In consequence, we systematically analyzed the pharmacological effects of RosA by using the method of network pharmacology. Firstly, the server of TCMSP was used to evaluate the biological characteristics of RosA. In addition, we predicted the potentially related target genes by chemical-gene interaction analysis. Furthermore, these potential target genes were used for GO enrichment analysis, KEGG enrichment analysis, and molecular docking. Finally, we systematically explained the potential targets and mechanism of RosA by constructing the pharmacological relationship network of RosA. Figure 1(b) is the workflow of RosA gene prediction and analysis process.

\section{Materials and Methods}

2.1. Screening Chemical Structure from PubChem Database. PubChem database (https://pubchem.ncbi.nlm.nih.gov/) is an open and accessible database resource, which contains important information resources such as drug discovery and chemical biology research $[13,14]$. We entered the keyword "rosmarinic acid" into the search box to retrieve the chemical formula (Figure 1(a)) and the CAS number of "RosA.".

2.2. Evaluation of Pharmacokinetics by TCMSP. TCMSP Database and Analysis Platform (http://lsp.nwu.edu.cn/ tcmsp.php) is one of the phytochemical databases. The database includes pharmacokinetic characteristics of natural compounds, such as oral bioavailability (OB), drug similarity (DL), blood-brain barrier (BBB), and water solubility [15]. Most of them can reflect the characteristics of drug absorption, distribution, metabolism, and excretion (ADME). Thus, in our study, the pharmacokinetic characteristics of RosA were searched and analyzed by the TCMSP database.

2.3. Screening of Gene Targets by the Comparative Toxicogenomics Database (CTD). CTD (http://ctdbase.org/) is a database that aims to clarify the relationship between genes, drugs, and diseases. It provides key information about the interrelation between genes, gene interactions, disease, and chemical phenotype. The information in the database is selected from peer-reviewed scientific literature $[16,17]$. The database was updated in 2019, which contains plenty of upto-date information [18]. The target genes and phenotypes related to specific compounds can be retrieved by CTD and ranked according to the interaction. Therefore, we used this database to predict RosA's target genes. Genes with interaction $\geq 1$ are selected as candidate target genes.

\subsection{Construction and Analysis of Protein-Protein Interaction} Network. The STRING 11.0 version (https://string-db.org/) is one of the online databases that can collect, score, and integrate all publicly available sources of information about protein-protein interactions. It can use computational predictions to supplement the existing information on protein-protein interactions. It achieves a comprehensive and objective global network, including direct (physical) and indirect (functional) interactions [19].

We uploaded 55 potential targets of RosA to the STRING database. The species was set as Homo sapiens and the minimum interaction score was 0.4 to build a protein interaction network. The results were imported into the Cytoscape3.7.2 version for visual analysis.

2.5. Gene Function and Pathway Enrichment Analysis. WebGestalt (http://www.webgestalt.org/option.php) is one of the popular software devices for functional and pathway enrichment analysis [20]. The GO is a comprehensive resource of the functions of genes and gene products containing molecular functions, biological pathways, and cellular components [21]. KEGG databases can give functional meanings to genes and genomes at the molecular levels and higher [22]. So, we input potential target genes into the WebGestalt server and use GO and KEGG databases for enrichment analysis.

2.6. Chemical Compound-Target-Pathway Network Construction. We have established a visual network through Cytoscape 3.7.2 version to further analyze and understand the complex relationships between RosA and targets and pathways.

2.7. Compound-Target Molecular Docking. Firstly, the chemical structure formula of RosA was downloaded from the CTD database and saved in mol2 format. Then, the $3 \mathrm{D}$ structures of target gene-associated proteins were downloaded from PDB data [23] (https://www.rcsb.org/) and saved in PDB format. The solvent molecules and ligands were removed by PyMOL software. AutoDock software was used to add hydrogenation, electron, and root and carry out other operations. And the formats of compounds and target proteins were converted to pdbqt format. During molecular docking, the protein structure was set as a rigid macromolecule, and the algorithm was Genetic Algorithm $\mathrm{Pa}$ rameters. For the group with the lowest binding energy of each protein in the results, PyMOL was used to draw 3D images, and one online tool (https://proteins.plus/) was used to generate $2 \mathrm{D}$ graphics.

\section{Results}

3.1. Molecular Formula and Pharmacokinetic Characteristics of RosA. Through the PubChem database, we obtained the chemical formula of RosA (Figure 1(a)). The CAS number is 20283-92-5. The database also provides some DepositorSupplied Synonyms such as 537-15-5 (CAS number), and rosmarinic acid. At the same time, we obtained 12 essential ADME-related data of RosA through TCMSP (Table 1). As we can see from Table $1, \mathrm{MW}$ is 360.34 , DL is $0.35, \mathrm{OB}$ is $1.38 \%, \mathrm{BBB}$ is $-1.24, \mathrm{~A} \log \mathrm{P}$ is 2.69 , and $\mathrm{RBN}$ is 7 .

3.2. Prediction of Targets Gene of RosA. After that, we obtained the potential target genes related to RosA through the CTD database. We entered "rosmarinic acid" and used the 


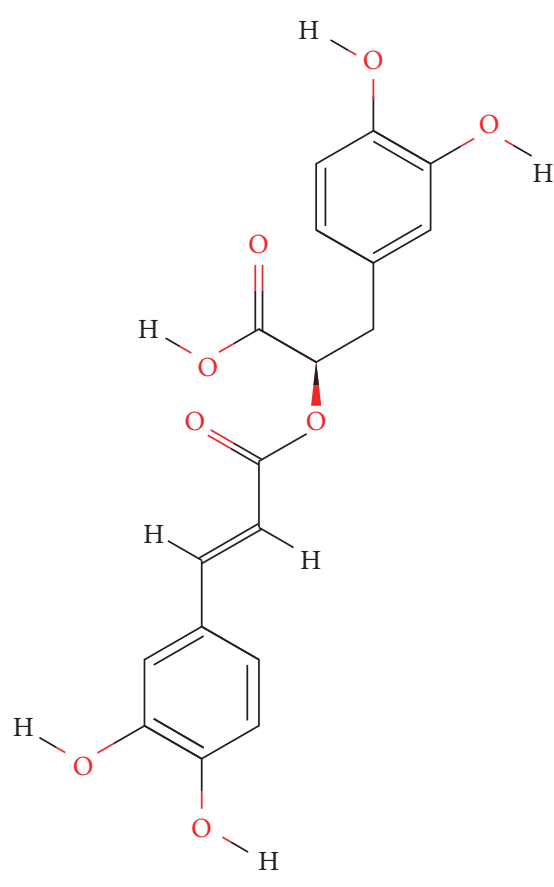

(a)

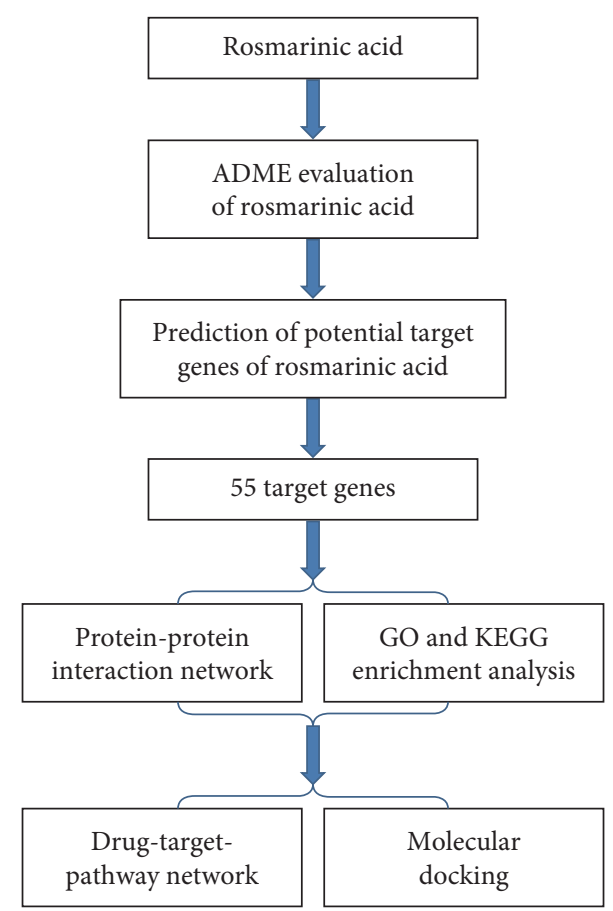

(b)

FIgUre 1: (a) Molecular structural formula of rosmarinic acid (PubChem CID: 5281792). (b) The flow of bioinformatics analysis of RosA includes target gene prediction, ADME evaluation, GO enrichment analysis, KEGG enrichment analysis, the construction of proteinprotein interaction network, the construction of the drug-target-pathway network, and molecular docking.

default settings as a screening condition to search candidate genes. Then, we obtained 55 target genes related to RosA (Table 2). These target genes were used for further analysis.

3.3. Protein-Protein Interaction Network. We imported the targets of RosA into the STRING database and selected organism as Homo sapiens to get the protein interaction network relationship. Then, we removed the free nodes and imported the results into Cytoscape 3.7.2 version for visualization (Figure 2). The size and color of the circle varied with the degree value. The width and color of edges varied with the combined score. There are 64 targets and 742 interactions in the picture. Calculated by the Network Analyzer plug-in in Cytoscape, the average degree value of the node is 23.19, the average Betweenness Centrality is 0.01 , and the average Closeness Centrality is 0.61. Among them, there are 18 nodes whose degree value, Betweenness Centrality, and Closeness Centrality are all greater than the average. They may be the main targets for RosA to play a role. The detailed node parameters are shown in Table 3.

3.4. GO Enrichment Analysis. In order to further analyze the 55 selected genes, we carried out GO enrichment analysis of these candidate genes by WebGestalt tool. GO enrichment analysis showed that most of all potential genes were involved in biological process (BP), cellular component (CC), and molecular function (MF). BP enrichment mainly contained the following target genes: biological regulation (52/55), response to stimulus (51/55), metabolic process (49/55), multicellular organismal process (47/55), cell communication (43/55), developmental
TABLe 1: Pharmacological and molecular properties data of RosA.

\begin{tabular}{|c|c|c|c|c|}
\hline Name & MW & AlogP & OB (\%) & Caco-2 \\
\hline
\end{tabular}

\begin{tabular}{llllllll}
\hline RosA & 360.34 & 2.69 & 1.38 & -0.54 & -1.24 & 0.35 & 7
\end{tabular}

MW, molecular weight; Caco-2, caco-2 permeability; OB, oral bioavailability; DL, drug-likeness; BBB, blood-brain barrier.

process (43/55), cellular component organization (38/55), localization (38/55), multiorganism process (34/55), and cell proliferation (26/55). CC enrichment was mainly involved in the following target genes: membrane-enclosed lumen (33/55), membrane (32/55), cytosol (32/55), vesicle (28/55), extracellular space (27/55), nucleus (27/55), endomembrane system (27/55), and protein-containing complex (21/55). MF enrichment was mainly engaged in the following target genes: protein binding (49/55) and ion binding (30/55). All the results are shown in Figure 3.

3.5. KEGG Enrichment Analysis. At the same time, we carried out KEGG enrichment analysis of these candidate genes by WebGestalt tool. KEGG pathway enrichment analysis displayed that 55 potential target genes were enriched and 120 signal pathways were significantly related with the target genes (FDR $\leq 0.05)$. In Figure 4, we show the top 20 pathways with the highest enrichment ratio.

3.6. Network Analysis. In order to further clearly show the relationship between compound (RosA), targets, and pathway clearly, we constructed a drug-target-pathway 
TABle 2: Target genes of RosA from CTD database.

\begin{tabular}{|c|c|c|c|}
\hline No. & Gene ID & Gene symbol & Description \\
\hline 1 & 10544 & PROCR & Protein $\mathrm{C}$ receptor \\
\hline 2 & 6868 & Adam 17 & Adam metallopeptidase domain 17 \\
\hline 3 & 5970 & RELA & RELA proto-oncogene, NF-kB subunit \\
\hline 4 & 7040 & TGFB1 & Transforming growth factor beta 1 \\
\hline 5 & 7124 & TNF & Tumor necrosis factor \\
\hline 6 & 3553 & IL1B & Interleukin 1 beta \\
\hline 7 & 3569 & IL6 & Interleukin 6 \\
\hline 8 & 5468 & PPARG & Peroxisome proliferator-activated receptor gamma \\
\hline 9 & 847 & CAT & Catalase 1 \\
\hline 10 & 4780 & NFE2L2 & Nuclear factor, erythroid 2 like 2 \\
\hline 11 & 1277 & COL1A1 & Collagen type I alpha 1 chain \\
\hline 12 & 1401 & CRP & C-reactive protein \\
\hline 13 & 2919 & CXCL1 & $\mathrm{C}-\mathrm{X}-\mathrm{C}$ motif chemokine ligand 1 \\
\hline 14 & 59 & ACTA2 & Actin alpha 2, smooth muscle \\
\hline 15 & 836 & CASP3 & Caspase 3 \\
\hline 16 & 1490 & CCN2 & Cellular communication network factor 2 \\
\hline 17 & 1281 & COL3A1 & Collagen type III alpha 1 chain \\
\hline 18 & 2876 & GPX1 & Glutathione peroxidase 1 \\
\hline 19 & 9446 & GSTO1 & Glutathione S-transferase omega 1 \\
\hline 20 & 3162 & HMOX1 & Heme oxygenase 1 \\
\hline 21 & 9817 & KEAP1 & Kelch-like ECH-associated protein 1 \\
\hline 22 & 4313 & MMP2 & Matrix metallopeptidase 2 \\
\hline 23 & 4318 & MMP9 & Matrix metallopeptidase 9 \\
\hline 24 & 4843 & NOS2 & Nitric oxide synthase 2 \\
\hline 25 & 1728 & NQO1 & $\mathrm{NAD}(\mathrm{P}) \mathrm{H}$-quinone dehydrogenase 1 \\
\hline 26 & 5054 & SERPINE1 & Serpin family $E$ member 1 \\
\hline 27 & 6647 & SOD1 & Superoxide dismutase 1 \\
\hline 28 & 6648 & SOD2 & Superoxide dismutase 2 \\
\hline 29 & 7498 & $\mathrm{XDH}$ & Xanthine dehydrogenase \\
\hline 30 & 9131 & AIFM1 & Apoptosis inducing factor mitochondria associated 1 \\
\hline 31 & 595 & CCND1 & Cyclin D1 \\
\hline 32 & 968 & CD68 & CD68 molecule \\
\hline 33 & 1312 & COMT & catechol-O-methyltransferase \\
\hline 34 & 1674 & DES & Desmin \\
\hline 35 & 1786 & DNMT1 & DNA methyltransferase 1 \\
\hline 36 & 2335 & FN1 & Fibronectin 1 \\
\hline 37 & 2353 & FOS & Fos proto-oncogene, AP-1 transcription factor subunit \\
\hline 38 & 2875 & GPT & Glutamic--pyruvic transaminase \\
\hline 39 & 3146 & HMGB1 & High mobility group box 1 \\
\hline 40 & 3725 & JUN & Jun proto-oncogene, AP-1 transcription factor subunit \\
\hline 41 & 157855 & KCNU1 & Potassium calcium-activated channel subfamily $U$ member 1 \\
\hline 42 & 4128 & MAOA & Monoamine oxidase A \\
\hline 43 & 4129 & MAOB & Monoamine oxidase B \\
\hline 44 & 6885 & MAP3K7 & Mitogen-activated protein kinase kinase kinase 7 \\
\hline 45 & 5594 & MAPK1 & Mitogen-activated protein kinase 1 \\
\hline 46 & 5595 & MAPK3 & Mitogen-activated protein kinase 3 \\
\hline 47 & 5599 & MAPK8 & Mitogen-activated protein kinase 8 \\
\hline 48 & 5601 & MAPK9 & Mitogen-activated protein kinase 9 \\
\hline 49 & 4353 & MPO & Myeloperoxidase \\
\hline 50 & 4615 & MYD88 & MYD88 innate immune signal transduction adaptor \\
\hline 51 & 4846 & NOS3 & Nitric oxide synthase 3 \\
\hline 52 & 4968 & OGG1 & 8-Oxoguanine DNA glycosylase \\
\hline 53 & 8505 & PARG & Poly (ADP-ribose) glycohydrolase \\
\hline 54 & 7099 & TLR4 & Toll-like receptor 4 \\
\hline 55 & 7431 & VIM & Vimentin \\
\hline
\end{tabular}

network diagram by Cytoscape 3.7.2 version. Figure 5 shows 176 nodes and 911 edges. Green circles represent compounds, yellow triangles correspond to targets, and red inverted triangles represent pathways.
3.7. Molecular Docking Results. It is generally believed that the lower the energy of the conformational stability of the ligand and the receptor, the greater the possibility of interaction. At present, there is no unified standard for target 


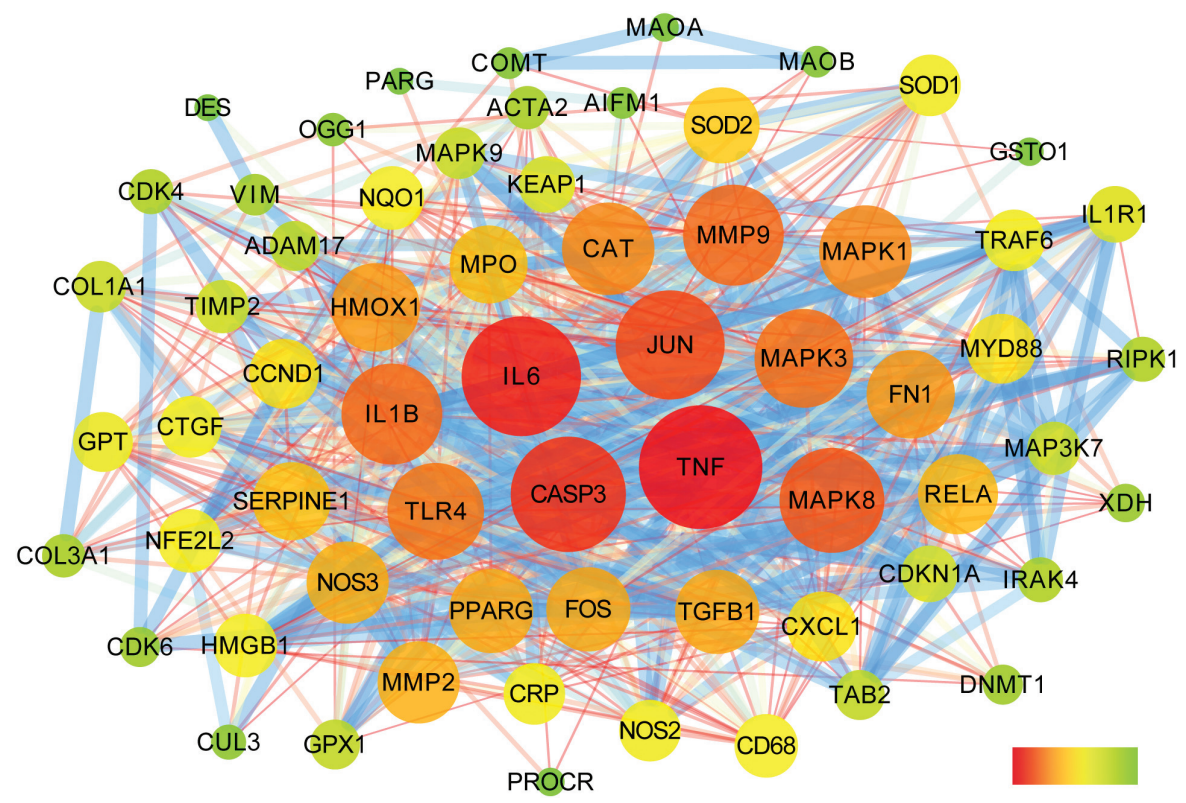

FIgURE 2: Protein interaction network of RosA target genes.

TABLE 3: Key protein topological parameters of protein interaction network.

\begin{tabular}{lccc}
\hline Name & Degree & Betweenness centrality & Closeness centrality \\
\hline TNF & 53 & 0.06924682 & 0.8630137 \\
IL6 & 51 & 0.05915435 & 0.84 \\
CASP3 & 49 & 0.08868983 & 0.81818182 \\
JUN & 46 & 0.03570932 & 0.7875 \\
MAPK8 & 44 & 0.02681408 & 0.76829268 \\
IL1B & 42 & 0.01914187 & 0.75 \\
MMP9 & 42 & 0.02381611 & 0.75 \\
MAPK3 & 41 & 0.01732055 & 0.74117647 \\
TLR4 & 40 & 0.01981721 & 0.73255814 \\
CAT & 38 & 0.07900048 & 0.71590909 \\
MAPK1 & 38 & 0.0134504 & 0.71590909 \\
HMOX1 & 36 & 0.01175721 & 0.7 \\
FN1 & 36 & 0.01346746 & 0.7 \\
FOS & 34 & 0.02316259 & 0.68478261 \\
TGFB1 & 34 & 0.03713298 & 0.68478261 \\
SERPINE1 & 31 & 0.01151125 & 0.66315789 \\
SOD2 & 30 & 0.03539503 & 0.64948454 \\
NQO1 & 24 & 0.01251491 & 0.61165049 \\
\hline
\end{tabular}

screening of active molecules. The active components with binding energy $\leq-5.0 \mathrm{~kJ} / \mathrm{mol}$ were selected as the basis for screening. The results of molecular docking showed that 13 of the selected target proteins had an affinity of less than $-5.0 \mathrm{~kJ} / \mathrm{mol}$ to RosA. The figure shows the best four docking results with the lowest binding energy. The results are shown in Table 4 and Figure 6.

\section{Discussion}

At present, the method of network pharmacology has been paid more and more attention during the process of drug development and utilization. This method can, firstly, evaluate, screen, and optimize some important characteristics of drugs, so as to speed up or simplify the process of drug discovery [11, 12]. Using this network analysis method, we not only obtained some important biological characteristics and some potentially related genes of RosA but also carried out GO and KEGG enrichment analysis.

Lipinski's Rule of Five is a rule of thumb. It is mainly used to evaluate whether a compound can be used as a drug or whether a compound with pharmacology or biological activity can become a human oral drug. Lipinski's rule of five mainly includes the following criteria: the molecular weight $(\mathrm{MW}) \leq 500 \mathrm{~g} / \mathrm{mol}$; an octanol-water partition coefficient log $\mathrm{P}(\log \mathrm{P}) \leq 5$; the donor of hydrogen bond (nitrogen or oxygen atoms with one or more hydrogen atoms) $\leq 5$; the number of hydrogen bond receptors (nitrogen or oxygen atoms $) \leq 10[24,25]$. The five rules are often referred to as guidelines for screening optimization of chemical libraries in the field of drug development [26]. At the same time, many studies have shown that the properties of compounds or drugs, such as molecular weight, lipophilicity, ionization, hydrogen bonding, polarity, and aromaticity, can affect their absorption, distribution, metabolism, excretion, and toxicity $[27,28]$. It can be seen from Figure 1 and Table 1 that the pharmacokinetic characteristics of RosA are basically consistent with Lipinski's Rule of Five. At the same time, several studies suggested that RosA is metabolized by the gut microbiota into caffeic acid and its derivatives after ingestion [29-31]. And a higher metabolic rate was observed in the liver than in the intestine [32]. Then the caffeic acid produced by RosA is absorbed, combined, and methylated in tissues such as the digestive tract and liver to produce various metabolites [29].

DL is used to evaluate the "drug-like" degree of expected compounds. This helps to optimize pharmacokinetics and drug properties (such as solubility and chemical stability). The DL level of a compound is $\geq 0.18$, which can be used as 


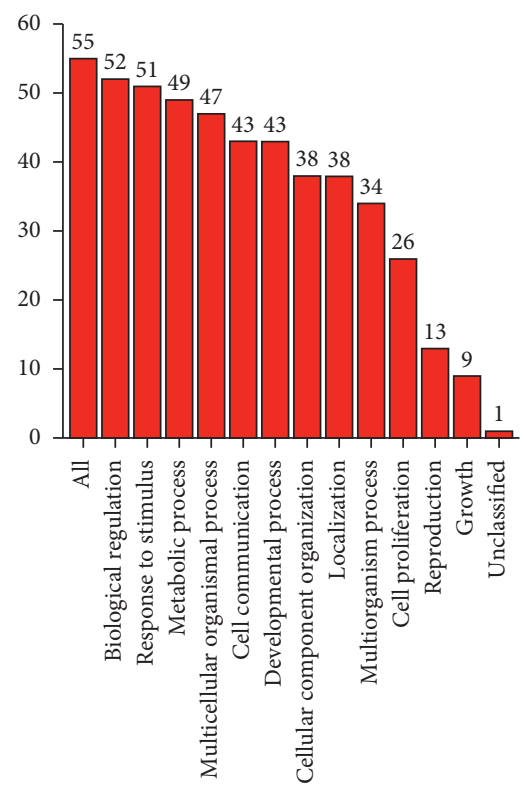

(a)

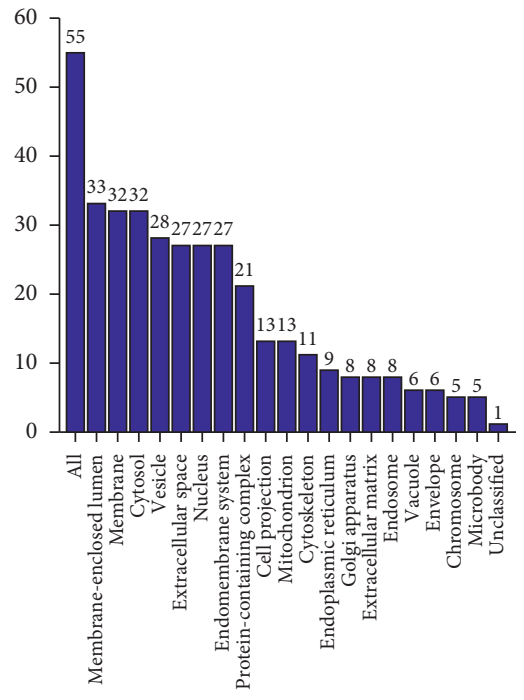

(b)

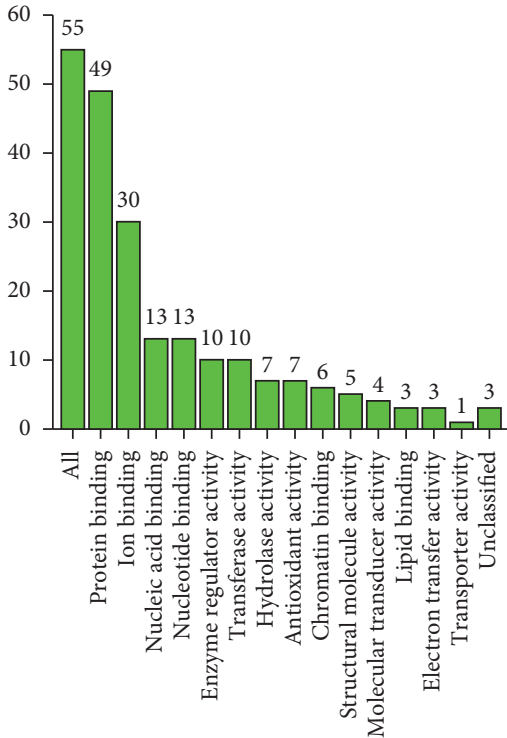

(c)

FIGURE 3: GO enrichment summary of the target genes. Biological process (BP), cellular component (CC), and molecular function (MF) are expressed by red (a), blue (b), and green (c), respectively. The height of the bar represents the number of target genes.

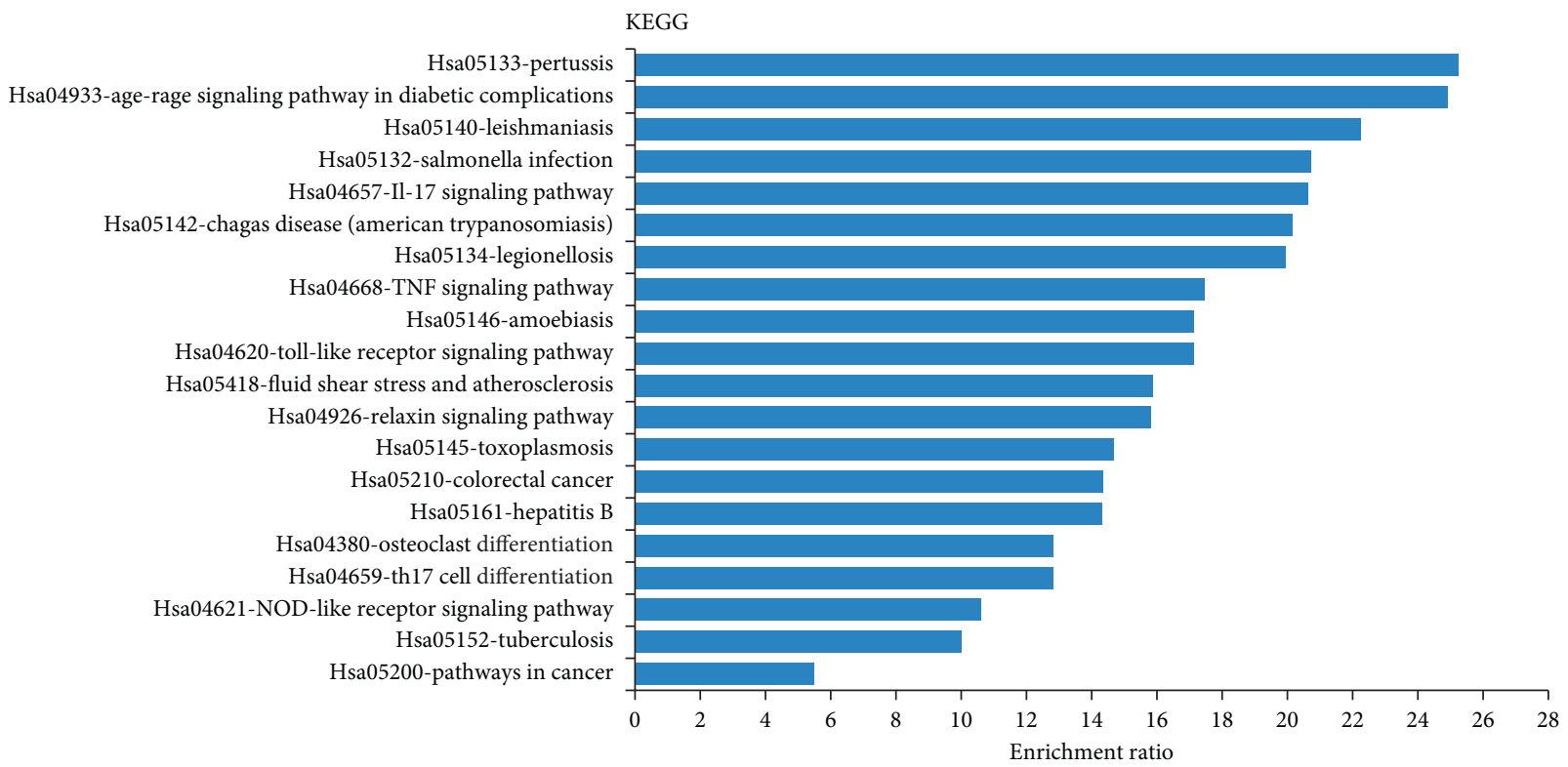

FIGURE 4: KEGG enrichment analysis of target genes.

one of the screening criteria of the traditional Chinese herbs [33]. The DL value of RosA is 0.35 . These all mean that RosA is one of the good candidates for drug development. However, the disadvantage of RosA is that its OB is relatively low. RBN are the number of bonds which allow free rotation around themselves. In particular, compounds which meet only the criteria of 10 or fewer rotatable bonds are predicted to have good oral bioavailability [34]. The RBN value of RosA is 7 , which means that RosA may have a good oral bioavailability. What is worse, it is not easy to cross the BBB. However, some studies suggested that drug utilization can be improved by improving the extraction method of RosA [32] or by nanocarriers $[35,36]$. But we still need to conduct further research and development on the practical application of RosA.

In the process of drug discovery, the search for target genes is a very important link. More and more molecules, compounds, and drugs have been found to have complex relationships with many genes and proteins [37-40]. At the same time, more and more online analysis tools have been developed in these studies. As shown in Table 2, 55 potential target genes related to RosA were identified by CTD database analysis. After that, we analyzed the 55 potential target genes through the STRING database. Degree value, 


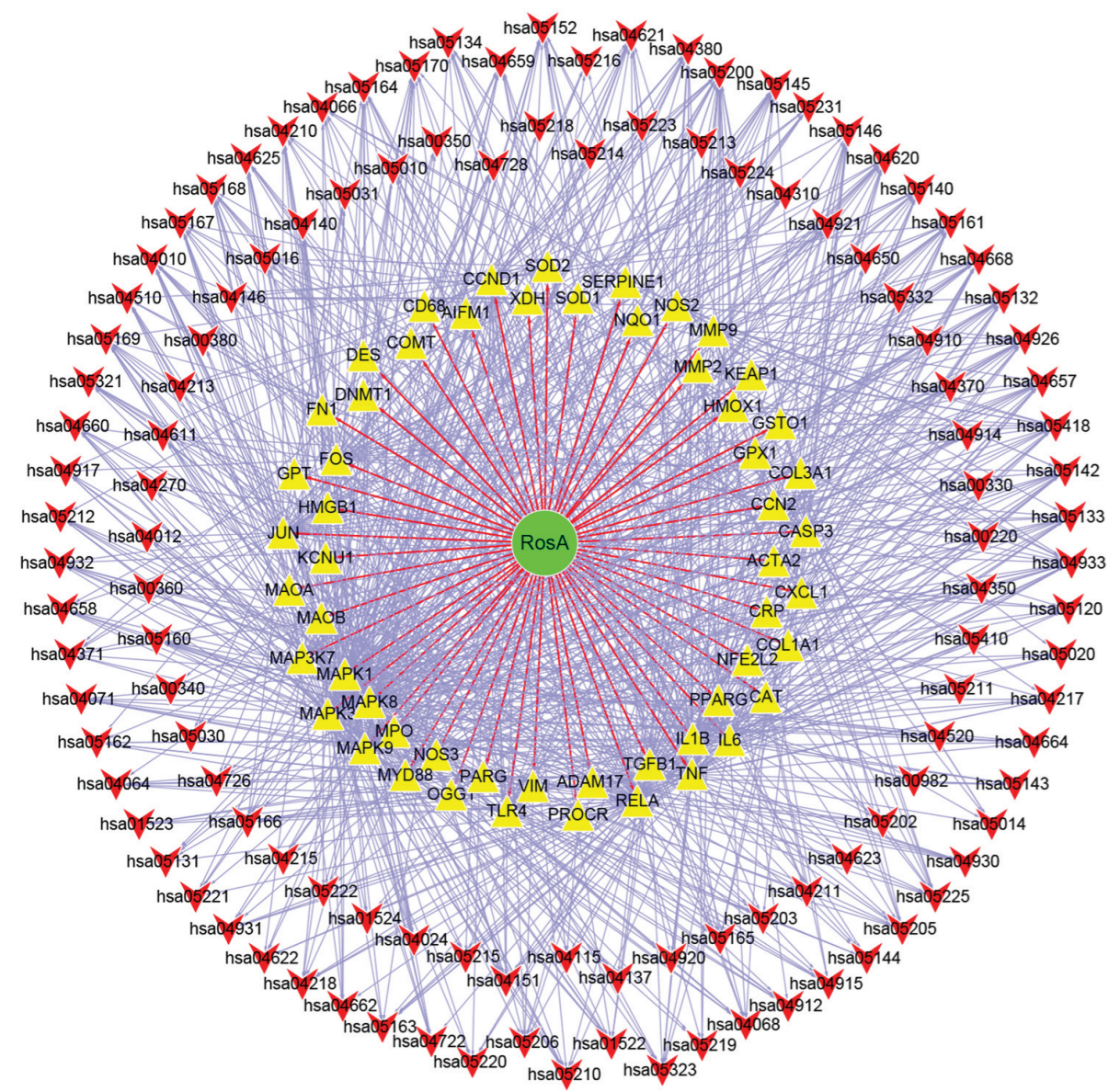

Figure 5: Compound (RosA)-target-pathway network.

TABLE 4: Compound-target molecular docking binding energy.

\begin{tabular}{lccc}
\hline No. & Targets & Compound & Binding energy $(\mathrm{kJ} / \mathrm{mol})$ \\
\hline 1 & MMP9 & RosA & -7.76 \\
2 & MAPK3 & & -7.71 \\
3 & JUN & & -6.83 \\
4 & CAT & -6.68 \\
5 & SERPINE1 & -6.61 \\
6 & MAPK8 & -6.16 \\
7 & FN1 & -6.09 \\
8 & FOS & -6.02 \\
9 & TGFB1 & -5.87 \\
10 & TNF & -5.73 \\
11 & MAPK1 & -5.57 \\
12 & TLR4 & -5.5 \\
13 & IL1B & -5.31 \\
14 & NQO1 & -4.76 \\
15 & CASP3 & -4.73 \\
16 & SOD2 & -4.72 \\
17 & HMOX1 & -4.34 \\
18 & IL6 & -3.55 \\
\hline
\end{tabular}

Betweenness Centrality, and Closeness Centrality are all greater than the average of 18 nodes, most of which (such as TNF, IL6, CASP3, JUN, MAPK8, IL1B, MMP9, MAPK3, and TLR4) participate in the process of immuneinflammatory response. At the same time, in order to further explore the possible interaction between RosA and these targets, we made a molecular docking. When the conformational energy of the ligand binding to the receptor is stable, the lower the energy is, the greater the possibility of interaction is. Taking the binding energy $\leq-5.0 \mathrm{~kJ} \cdot \mathrm{mol}^{-1}$ as the screening criterion, we found that the binding energy of 13 of the 18 targets was less than $-5.0 \mathrm{~kJ} \cdot \mathrm{mol}^{-1}$; that is, RosA may interact directly with these targets. So, they may be the key targets for the pharmacological action of RosA.

We did further GO analysis and pathway analysis of these potential genes through WebGestalt. According to the analysis of BP items, RosA is closely related to biological regulation, response to stimulus, metabolic process, multicellular organismal process, cell communication, developmental process, and so on. These results indicate that RosA may be related to anti-infection and antitumor. What is more, 9 of the first 20 enriched KEGG pathways are related to bacterial, viral, and parasite infections, such as pertussis, Chagas disease (American trypanosomiasis), salmonella infection, hepatitis B, amoebiasis, and toxoplasmosis. These results suggested that RosA may have antibacterial, antiviral, and anti-infective effects. Some of these pathways are related to inflammatory diseases and tumor diseases, for example, the AGE-RAGE signaling pathway in diabetic complications, colorectal cancer. Other studies suggested that RosA can inhibit the growth of cancer cells [41-43]. In addition, 


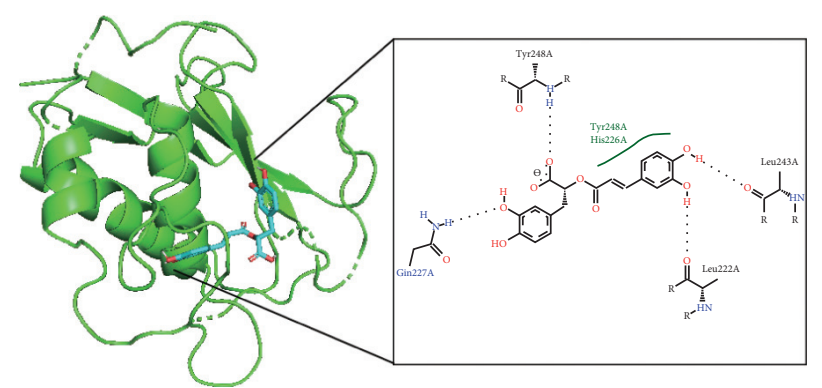

(a)

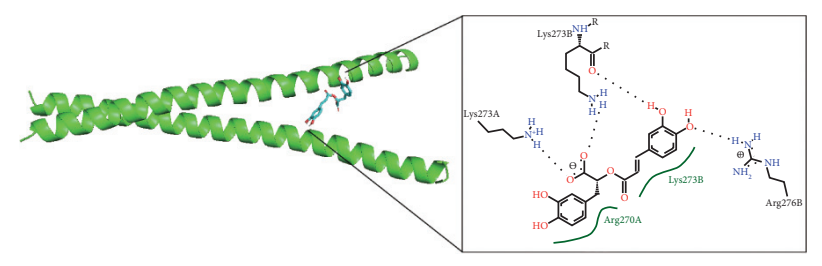

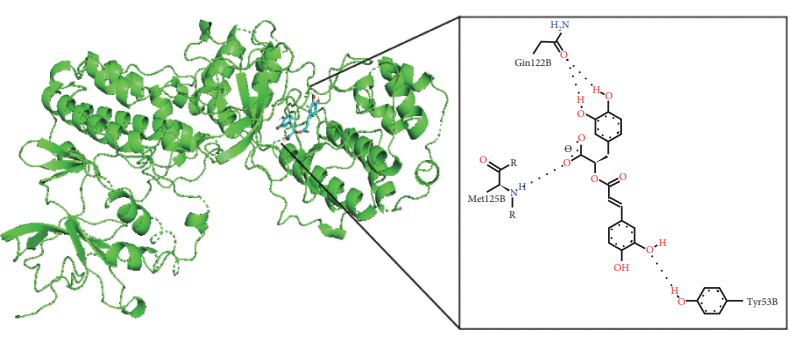

(b)

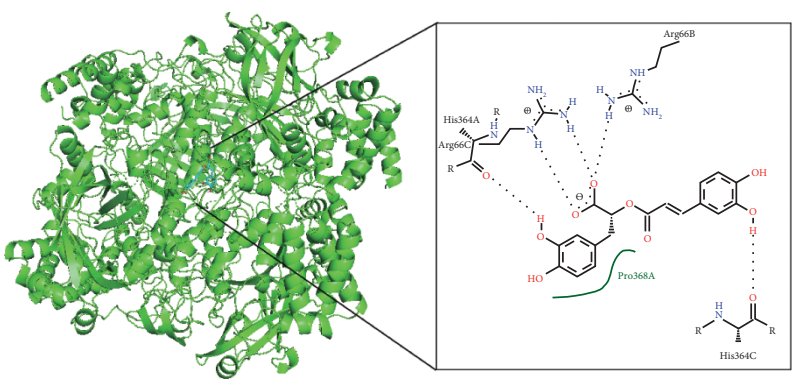

(d)

Figure 6: 3D and 2D pictures of the four best docking results. (a) MMP9 and RosA; (b) MAPK3 and RosA; (c) JUN and RosA; (d) CAT and RosA.

KEGG enrichment results also include IL-17 signaling pathway, TNF signaling pathway, Toll-like receptor signaling pathway, NOD-like receptor signaling pathway, Th17 cell differentiation, relaxing signaling pathway, and other immunoinflammatory response-related pathways. Therefore, our study suggested that RosA may play a role by inhibiting excessive immune response, storm of inflammatory factors, and proliferation of tumor cells. Wei et al. suggested that RosA can reduce inflammation and atherosclerosis $[44,45]$. Similarly, studies showed that RosA can also inhibit allergic inflammation in mice [46-48]. This is similar to our GO and KEGG analysis results. What is more, we made a drug-target network diagram, which further indicates that RosA may have a wide range of pharmacological activities.

\section{Conclusions}

Our studies showed that RosA has many pharmacological activities. At the same time, we analyzed the possible mechanism of action of RosA, which can be used to further develop safe and effective anti-inflammatory and anticancer drugs. Our work provides a new idea for the research, development, and clinical application of RosA. However, its shortcomings such as low oral availability and not easy to pass through the $\mathrm{BBB}$ also bring new challenges.

\section{Data Availability}

The data used to support the findings of this study are included within the article.

\section{Conflicts of Interest}

The authors declare that they have no conflicts of interest.

\section{Authors' Contributions}

Minglong Guan collected and analyzed all the data and is the major writer of the paper. Lan Guo helped in writing the paper and contributed to the analysis of these data. Hengli $\mathrm{Ma}$ and Huimei Wu revised the paper. Minglong Guan and Xiaoyun Fan designed the study. Xiaoyun Fan also revised the paper.

\section{Acknowledgments}

This work was supported by grants from the National Natural Science Foundation of China (Grant nos. U1803126 and 81770032).

\section{References}

[1] B. E. Ellis and G. H. N. Towers, "Biogenesis of rosmarinic acid in Mentha," Biochemical Journal, vol. 118, no. 2, pp. 291-297, 1970.

[2] F. Yuan, R. Liu, M. Hu et al., "JAX2, an ethanol extract of Hyssopus cuspidatus Boriss, can prevent bronchial asthma by inhibiting MAPK/NF- $\kappa$ B inflammatory signaling," Phytomedicine, vol. 57, pp. 305-314, 2019.

[3] W. Boonyarikpunchai, S. Sukrong, and P. Towiwat, "Antinociceptive and anti-inflammatory effects of rosmarinic acid isolated from Thunbergia laurifolia Lindl," Pharmacology Biochemistry and Behavior, vol. 124, pp. 67-73, 2014.

[4] W. Cao, C. Hu, L. Wu, L. Xu, and W. Jiang, "Rosmarinic acid inhibits inflammation and angiogenesis of hepatocellular carcinoma by suppression of NF- $\kappa \mathrm{B}$ signaling in $\mathrm{H} 22$ tumorbearing mice," Journal of Pharmacological Sciences, vol. 132, no. 2, pp. 131-137, 2016.

[5] Y.-C. Chung, F.-C. Hsieh, Y.-J. Lin et al., "Magnesium lithospermate $\mathrm{B}$ and rosmarinic acid, two compounds present in 
Salvia miltiorrhiza, have potent antiviral activity against enterovirus 71 infections," European Journal of Pharmacology, vol. 755, pp. 127-133, 2015.

[6] M. Ghasemzadeh Rahbardar, B. Amin, S. Mehri, S. J. MirnajafiZadeh, and H. Hosseinzadeh, "Anti-inflammatory effects of ethanolic extract of Rosmarinus officinalis L. and rosmarinic acid in a rat model of neuropathic pain," Biomedicine \& Pharmacotherapy, vol. 86, pp. 441-449, 2017.

[7] Y. Tsukamoto, S. Ikeda, K. Uwai et al., "Rosmarinic acid is a novel inhibitor for Hepatitis B virus replication targeting viral epsilon RNA-polymerase interaction," PLoS One, vol. 13, Article ID e197664, 2018.

[8] H.-A. Oh, C.-S. Park, H.-J. Ahn, Y. S. Park, and H.-M. Kim, "Effect of Perilla frutescens var. acuta Kudo and rosmarinic acid on allergic inflammatory reactions," Experimental Biology and Medicine, vol. 236, no. 1, pp. 99-106, 2011.

[9] R. Domitrović, M. Škoda, V. Vasiljev Marchesi et al., "Rosmarinic acid ameliorates acute liver damage and fibrogenesis in carbon tetrachloride-intoxicated mice," Food and Chemical Toxicology, vol. 51, pp. 370-378, 2013.

[10] K. Lou, R. Zhang, and W. Hu, "Rosmarinic acid stimulates liver regeneration through the mTOR pathway," Phytomedicine, vol. 23, pp. 1-22, 2016.

[11] Y.-H. Bi, L.-h. Zhang, S.-j. Chen, and Q.-z. Ling, "Antitumor mechanisms of curcumae rhizoma based on network pharmacology," Evidence-based Complementary and Alternative Medicine, vol. 2018, 9 pages, 2018.

[12] Y. Hu and D. Chen, "Analysis of the action mechanism of Fang Ji Huang Qi decoction in treating rheumatoid arthritis by network pharmacology," Traditional Medicine Research, vol. 3, pp. 286-294, 2018.

[13] Y. Wang, S. H. Bryant, T. Cheng et al., "PubChem BioAssay: 2017 update," Nucleic Acids Research, vol. 45, no. D1, pp. D955-D963, 2017.

[14] S. Kim, J. Chen, T. Cheng et al., "PubChem 2019 update: improved access to chemical data," NUCLEIC ACIDS RESEARCH, vol. 47, no. D1, pp. D1102-D1109, 2019.

[15] J. Ru, P. Li, J. Wang et al., "TCMSP: a database of systems pharmacology for drug discovery from herbal medicines," Journal of Cheminformatics, vol. 6, pp. 1-6, 2014.

[16] A. P. Davis, C. J. Grondin, K. Lennon-Hopkins et al., "The Comparative Toxicogenomics Database's 10th year anniversary: update 2015," Nucleic Acids Research, vol. 43, no. D1, pp. D914-D920, 2015.

[17] A. P. Davis, C. J. Grondin, R. J. Johnson et al., "The comparative Toxicogenomics database: update 2017," Nucleic Acids Research, vol. 45, no. D1, pp. D972-D978, 2017.

[18] A. P. Davis, C. J. Grondin, R. J. Johnson et al., "The comparative Toxicogenomics database: update 2019," Nucleic Acids Research, vol. 47, no. D1, pp. D948-D954, 2019.

[19] D. Szklarczyk, A. L. Gable, D. Lyon et al., "STRING v11: proteinprotein association networks with increased coverage, supporting functional discovery in genome-wide experimental datasets," Nucleic Acids Research, vol. 47, no. D1, pp. D607-D613, 2019.

[20] J. Wang, D. Duncan, Z. Shi, and B. Zhang, "WEB-based GEne SeT AnaLysis toolkit (WebGestalt): update 2013," Nucleic Acids Research, vol. 41, no. W1, pp. W77-W83, 2013.

[21] The Gene Ontology Consortium, "Expansion of the gene Ontology knowledgebase and resources," Nucleic Acids Research, vol. 45, pp. D331-D338, 2017.

[22] M. Kanehisa, M. Furumichi, M. Tanabe, Y. Sato, and K. Morishima, "KEGG: new perspectives on genomes, pathways, diseases and drugs," Nucleic Acids Research, vol. 45, no. D1, pp. D353-D361, 2017.
[23] S. K. Burley, C. Bhikadiya, C. Bi et al., "RCSB Protein Data Bank: powerful new tools for exploring 3D structures of biological macromolecules for basic and applied research and education in fundamental biology, biomedicine, biotechnology, bioengineering and energy sciences," Nucleic Acids Research, vol. 49, no. D1, pp. D437-D451, 2021.

[24] C. A. Lipinski, F. Lombardo, B. W. Dominy, and P. J. Feeney, "Experimental and computational approaches to estimate solubility and permeability in drug discovery and development settings 1PII of original article: S0169-409X(96)00423-1. The article was originally published in Advanced Drug Delivery Reviews 23 (1997) 3-25. 1," Advanced Drug Delivery Reviews, vol. 46, no. 1-3, pp. 3-26, 2001.

[25] C. A. Lipinski, "Lead- and drug-like compounds: the rule-offive revolution," Drug Discovery Today: Technologies, vol. 1, no. 4, pp. 337-341, 2004.

[26] P. D. Leeson, "Molecular inflation, attrition and the rule of five," Advanced Drug Delivery Reviews, vol. 101, pp. 22-33, 2016.

[27] M. P. Gleeson, A. Hersey, D. Montanari, and J. Overington, "Probing the links between in vitro potency, ADMET and physicochemical parameters," Nature Reviews Drug Discovery, vol. 10, no. 3, pp. 197-208, 2011.

[28] E. A. G. Blomme and Y. Will, "Toxicology strategies for drug discovery: present and future," Chemical Research in Toxicology, vol. 29, no. 4, pp. 473-504, 2016.

[29] S. Baba, N. Osakabe, M. Natsume et al., "Absorption, metabolism, degradation and urinary excretion of rosmarinic acid after intake of Perilla frutescens extract in humans," European Journal of Nutrition, vol. 44, no. 1, pp. 1-9, 2005.

[30] G. Williamson, A. J. Day, G. W. Plumb, and D. Couteau, "Human metabolic pathways of dietary flavonoids and cinnamates," Biochemical Society Transactions, vol. 28, no. 2, p. $16,2000$.

[31] A. R. Rechner, G. Kuhnle, P. Bremner, G. P. Hubbard, K. P. Moore, and C. A. Rice-Evans, "The metabolic fate of dietary polyphenols in humans," Free Radical Biology and Medicine, vol. 33, no. 2, pp. 220-235, 2002.

[32] M. Achour, S. Saguem, B. Sarriá, L. Bravo, and R. Mateos, "Bioavailability and metabolism of rosemary infusion polyphenols using Caco-2 and HepG2 cell model systems," Journal of The Science of Food and Agriculture, vol. 98, no. 10, pp. 3741-3751, 2018.

[33] W. Tao, X. Xue, W. Xia et al., "Network pharmacology-based prediction of the active ingredients and potential targets of Chinese herbal Radix Curcumae formula for application to cardiovascular disease," Journal of Ethnopharmacology, vol. 145, no. 1, pp. 1-10, 2013.

[34] D. F. Veber, S. R. Johnson, H.-Y. Cheng, B. R. Smith, K. W. Ward, and K. D. Kopple, "Molecular properties that influence the oral bioavailability of drug candidates," Journal of Medicinal Chemistry, vol. 45, no. 12, pp. 2615-2623, 2002.

[35] R. Bhatt, D. Singh, A. Prakash et al., "Development, characterization and nasal delivery of rosmarinic acid-loaded solid lipid nanoparticles for the effective management of Huntington's disease," Drug Delivery, vol. 22, no. 7, pp. 931-939, 2013.

[36] S. B. Da Silva, M. Amorim, P. Fonte et al., "Natural extracts into chitosan nanocarriers for rosmarinic acid drug delivery," Pharmaceutical Biology, vol. 53, no. 5, pp. 642-652, 2014.

[37] L. Wang and X.-Q. Xie, "Computational target fishing: what should chemogenomics researchers expect for the future ofin silicodrug design and discovery?" Future Medicinal Chemistry, vol. 6, no. 3, pp. 247-249, 2014. 
[38] A. L. Hopkins, "Network pharmacology: the next paradigm in drug discovery," Nature Chemical Biology, vol. 4, no. 11, pp. 682-690, 2008.

[39] J. Wang, L. Gao, and Y. M. Lee, "Target identification of natural and traditional medicines with quantitative chemical proteomics approaches," Pharmacology and Therapeutics, vol. 162, pp. 10-22, 2016.

[40] M. Schenone, V. Dančík, B. K. Wagner, and P. A. Clemons, "Target identification and mechanism of action in chemical biology and drug discovery," Nature Chemical Biology, vol. 9, no. 4, pp. 232-240, 2013.

[41] Y. Zhang, H. Min, L. Liu et al., "Anticancer effects of Rosmarinic acid in OVCAR-3 ovarian cancer cells are mediated via induction of apoptosis, suppres- sion of cell migration and modulation of lncRNA MALAT-1 expression," Journal of Balkan Union of Oncology, vol. 23, no. 3, pp. 763-768, 2018.

[42] I. Radziejewska, K. Supruniuk, J. Nazaruk et al., "Rosmarinic acid influences collagen, MMPs, TIMPs, glycosylation and MUC1 in CRL-1739 gastric cancer cell line," Biomedicine \& Pharmacotherapy, vol. 107, pp. 397-407, 2018.

[43] Y.-G. Jang, K.-A. Hwang, and K.-C. Choi, "Rosmarinic acid, a component of rosemary tea, induced the cell cycle arrest and apoptosis through modulation of HDAC2 expression in prostate cancer cell lines," Nutrients, vol. 10, no. 11, p. 1784, 2018.

[44] Y. Wei, J. Chen, Y. Hu et al., "Rosmarinic acid mitigates lipopolysaccharide-induced neuroinflammatory responses through the inhibition of TLR4 and CD14 expression and NF$\kappa \mathrm{B}$ and NLRP3 inflammasome activation," Inflammation, vol. 41, no. 2, pp. 732-740, 2018.

[45] Y. Yao, J. Mao, S. Xu et al., "Rosmarinic acid inhibits nicotineinduced C-reactive protein generation by inhibiting NLRP3 inflammasome activation in smooth muscle cells," Journal of Cellular Physiology, vol. 234, no. 2, pp. 1758-1767, 2019.

[46] Z. Liang, Y. Xu, X. Wen et al., "Rosmarinic acid attenuates airway inflammation and hyperresponsiveness in a murine model of asthma," Molecules, vol. 21, no. 6, p. 769, 2016.

[47] Z. Liang, H. Nie, Y. Xu et al., "Therapeutic effects of rosmarinic acid on airway responses in a murine model of asthma," International Immunopharmacology, vol. 41, pp. 90-97, 2016.

[48] F. Shakeri, N. Eftekhar, N. M. Roshan, R. Rezaee, A. Moghimi, and M. H. Boskabady, "Rosmarinic acid affects immunological and inflammatory mediator levels and restores lung pathological features in asthmatic rats," Allergologia Et Immunopathologia, vol. 47, no. 1, pp. 16-23, 2019. 